\title{
La representación social de la inseguridad en jóvenes universitarios ecuatorianos: el caso Ambato
}

\author{
Verónica Molina-Coloma, ${ }^{\star}$ Hiram Reyes-Sosa ${ }^{\star *}$, Maider Larrañaga-Egilegor ${ }^{\star * \star}$
}

Máster en Psicología. Profesora, programa de Psicología Clínica, Universidad Técnica de Ambato, Ambato, Ecuador.

Correo electrónico:

va.molina@uta.edu.ec

** Máster en Psicología. Doctorando en Psicología, Universidad del País Vasco/ Euskal Herriko Unibertsitatea, campus Gipuskoa, España.

Correo electrónico:

hiramreyez@gmail.com

*** Doctora en Psicología social. Profesora, Facultad de Psicología, Universidad del País Vasco/Euskal Herriko Unibertsitatea, campus Gipuskoa, España.

Correo electrónico:

maider.larranaga@ehu.es

Recibido: 17 de septiembre del 2015

Aprobado: 12 de noviembre del 2015

Cómo citar este artículo: Molina-Coloma,

V.; Reyes-Sosa, H. y Lagarraña-Egilegor,

M. (2015). La representación social de

la inseguridad en jóvenes universitarios

ecuatorianos: el caso Ambato. Pensando

Psicología, 11(18), 85-95. doi: http://dx.doi.

org/10.16925/pe.v11i18.1221

\section{Resumen}

Introducción: la inseguridad es un tema de preocupación social en Ecuador, así como en muchos países latinoamericanos. La referencia a sucesos relacionados con la inseguridad es habitual en los medios de comunicación y refleja la situación de incertidumbre con la que vive la población cotidianamente. Objetivo: se plantea el análisis de la representación social de la inseguridad. En este sentido, se pretende conocer cómo los jóvenes conciben y explican este objeto social. Metodología: mediante técnicas cualitativas, se realizó la recogida de información. En el estudio, participaron 271 jóvenes universitarios (67\% mujeres y 33\% hombres), con una media de 21,32 años (DT: 2,23). Resultados: el análisis del núcleo central y del sistema periférico reveló que los jóvenes perciben un contexto inseguro caracterizado por la incertidumbre y el miedo de salir a lugares públicos o zonas específicas. Este sentimiento de inseguridad se ve reforzado por la falta de mecanismos que regulen este clima negativo, tal como lo es la ineficiente estructura en las instituciones judiciales. En relación con las diferencias de género, se evidenció que las mujeres reportan tener mayor miedo y vulnerabilidad en su vida cotidiana, a diferencia de los hombres. Conclusiones: en Ambato se han desarrollado estrategias a fin de reducir la inseguridad, sin embargo, estos mecanismos no han ayudado a disminuir la percepción de miedo establecido en el contexto.

Palabras clave: género, incertidumbre, inseguridad, miedo, representaciones sociales. 


\title{
Social Representation of Insecurity among Young Ecuadorian University Students: the Ambato Case
}

\begin{abstract}
Introduction: Insecurity is a matter for social concern in Ecuador, as well as in many Latin American countries. References to events associated with insecurity are habitual in the communications media and reflect the situation of uncertainty experienced by the population on a daily basis. Objective: An analysis is proposed of the social representation of insecurity. The aim is thus to determine how young people conceive of and explain this social object. Methodology: Information was gathered using qualitative techniques. 271 young university students took part in the study (67\% women and 33\% men), with a median age of 21.32 (DT: 2.23). Results: Analysis of the central nucleus and peripheral system showed that young people perceive an insecure context characterized by uncertainty and fear of going out to public places or specific zones. This feeling of insecurity is further heightened by the lack of mechanisms to regulate this negative climate, such as the inefficient structure of judicial institutions. In relation to gender differences, it was found that women report experiencing greater fear and vulnerability in their daily lives than men. Conclusions: In Ambato, strategies have been developed to reduce insecurity; however, these mechanisms have not helped to decrease the perception of fear established in the context.
\end{abstract}

Keywords: gender, uncertainty, insecurity, fear, social representations.

\section{A representação social da insegurança em jovens universitários equatorianos: o caso Ambato}

\section{Resumo}

Introdução: a insegurança é um tema de preocupação social no Equador e em muitos países latino-americanos. A referência a acontecimentos relacionados com a insegurança é habitual nos meios de comunicação e reflete a situação de incerteza com a qual a população vive cotidianamente. Objetivo: apresenta-se a análise da representação social da insegurança. Nesse sentido, pretende-se conhecer como os jovens concebem e explicam esse objeto social. Metodologia: mediante técnicas qualitativas, realizou-se a coleta de informação. Do estudo, participaram 271 jovens universitários (67\% mulheres e 33\% homens), com uma média de 21,32 anos (DT: 2,23). Resultados: a análise do núcleo central e do sistema periférico revelou que os jovens percebem um contexto inseguro caracterizado pela incerteza e o medo de ir a lugares públicos ou áreas específicas. Esse sentimento de insegurança se vê reforçado pela falta de mecanismos que regulamentem esse clima negativo e pela ineficiente estrutura nas instituições judiciais. A respeito das diferenças de gênero, evidenciou-se que as mulheres relatam ter mais medo e vulnerabilidade em sua vida cotidiana do que os homens. Conclusões: em Ambato, vêm sendo desenvolvidas estratégias a fim de reduzir a insegurança; contudo, esses mecanismos não têm ajudado a diminuir a percepção de medo estabelecida no contexto.

Palavras-chave: gênero, incerteza, insegurança, medo, representações sociais. 


\section{Introducción}

La inseguridad es un fenómeno que ha fragmentado las relaciones interpersonales en Ecuador. Los hechos delictivos, con el paso del tiempo, han provocado una reducción de posibilidades en la socialización. En este sentido, el desconocido se convierte en un potencial delincuente y provoca que las personas dejen de salir a ciertas horas o que se ayude a extraños (Carrión, 1994). Además, ciertos lugares tales como parques, paradas de buses, transportes públicos o mercados, se convierten en lugares peligrosos. La inseguridad provoca que las personas pertenecientes a una sociedad desarrollen conductas individualistas, así como un sentimiento de desconfianza que los acompaña toda su vida (Carrión, 1994). En un contexto inestable y en el cual las instituciones gubernamentales (especialmente judiciales) carecen de eficacia, es comprensible el aumento de la seguridad privada, de manera que la seguridad se convierte en un privilegio más que en un derecho. Sin embargo, este aumento de la seguridad privada no conlleva a la disminución de la inseguridad, pero sí genera otras consecuencias como, por ejemplo, el desinterés por parte de las instituciones públicas hacia la protección ciudadana.

En este sentido, este estudio pretendió conocer cómo los jóvenes ambateños conciben y explican la inseguridad. Para ello, se plantea un acercamiento a la escuela estructural de la teoría de las representaciones sociales (TRS), ya que a través de su hipótesis del núcleo central es posible identificar los elementos que mayor importancia tienen al representar un objeto social, así como las relaciones que se establecen entre estos elementos y su actualización (Abric, 2001).

\section{La inseguridad en Ecuador}

La situación política, económica y social de Ecuador en las últimas décadas se ha caracterizado por una clara inestabilidad. Sin embargo, siempre se ha presentado como un país tranquilo en comparación con sus vecinos Perú y Colombia, lugares donde la violencia ha sido más visible. La realidad cotidiana durante muchos años en Ecuador ha sido diferente a lo que se proyectaba hacia afuera y hacia dentro. No obstante, los estudios de seguridad ciudadana han proporcionado datos que contradicen la percepción de seguridad que los gobiernos intentaban promover (Palomeque, 2002). En Ecuador, los altos índices delincuenciales aparecen ya en la década de los noventa, después del establecimiento de las políticas sobre ajuste estructural (Núñez, 2011). Los estudios realizados por el Observatorio de la Ciudad (Organización de la Sociedad Civil de Quito), muestran que desde 1990 hasta el 2000 la tasa de homicidios se duplicó, en concreto de 7 a 14 casos anuales por cada 100000 habitantes (Jarrín, 2005).

Entre 2001 y 2003, con el aumento de la inseguridad se incrementaron también las tasas de violencia. Los delitos en estos años crecieron exponencialmente. Según la Dirección Nacional de la Policía Judicial (Jarrín, 2005), los casos que mayor presencia y aumento tuvieron fueron las extorsiones con $106 \%$, los asaltos a bancos $69 \%$, los secuestros $41 \%$, las estafas $24 \%$, los homicidios $19 \%$ y las violaciones sexuales $60 \%$. Posteriormente, entre 2012 y 2013, se detectó que los delitos de mayor incidencia fueron el robo a personas, el robo a domicilios, el robo a vehículos, el robo en locales comerciales, la sustracción de accesorios de vehículos, homicidios-asesinatos, violaciones y muertes ocasionadas por accidentes de tráfico (Centro Ecuatoriano de Análisis de Seguridad Integral y Comisión de Estadística de Seguridad Integral, 2013). En este sentido, y gracias al establecimiento de un clima negativo, se puede entender que el $31 \%$ de los ciudadanos ecuatorianos indicaron que el principal problema del país es la inseguridad, y el $49 \%$ se preocupa por ser víctima de un delito (Latinobatómetro, 2013). En relación con la confianza institucional, el 52\% de los ciudadanos ha considerado que la institución que debe encargarse de velar por la seguridad es la policía nacional. Sin embargo, el 4,6\% de los ciudadanos considera que dicha institución no es efectiva en la resolución del problema de inseguridad. Estos datos evidencian la poca confianza institucional. En este sentido, un $29 \%$ de los ciudadanos indicaron que la razón por la cual no denunciaban los delitos era la falta de confianza en el sistema judicial (Instituto Nacional de Estadística y Censos, 2011).

Por su parte, y centrándose en la provincia de Tungurahua (Ambato), los ciudadanos señalan que el problema de la inseguridad va en aumento. Según la Fiscalía Provincial de Tungurahua, los cinco tipos de delitos con mayor presencia son los delitos denominados "contra el derecho a la propiedad". En el periodo 2011-2013, los índices anuales que caracterizan estos delitos fueron: robo simple con 1461 denuncias; hurto simple con 525 denuncias; sustracción sin violencia (con fraude en lugares públicos o concurridos) con 404 denuncias; estafa 329 denuncias; y robo 
agravado con 190 denuncias (Garcés, Martínez, Tite y Tamayo, 2014). Así, un 78\% de los ciudadanos señalaron que se sienten más inseguros en su ciudad, y un $46 \%$ señalaron que se sienten más inseguros en su barrio. Además, un $44 \%$ señaló un aumento de la delincuencia en su barrio, y un 79\% en la ciudad (INEC, 2011). En medio de este clima de inestabilidad se destaca el grupo de los jóvenes. A través de la Primera Encuesta Nacional sobre Jóvenes y Participación Política en Ecuador, se encontró que es este grupo el más afectado en relación con temas de inseguridad (10,32\%) y delincuencia (52\%). Además, de acuerdo con esta encuesta, se encontró también que desde la perspectiva de los jóvenes, la inseguridad y la delincuencia son las dos problemáticas que más los afectan (Ramírez y Ágora Democrática, 2011). Todos estos datos muestran la complejidad del fenómeno de la inseguridad y destacan el particular interés por ahondar en el mismo. Además, el interés que tiene el trabajar con el grupo de jóvenes, ya que es el grupo más vulnerable en relación con la inseguridad. Por lo tanto, conocer cómo en la actualidad los jóvenes perciben y explican su contexto puede ayudar a comprender mejor los factores y causas que promueven el aumento y establecimiento de este fenómeno social.

\section{Contribuciones de la teoría de las representaciones sociales al estudio de la inseguridad}

El estudio de la inseguridad mediante el enfoque de la TRS arroja resultados interesantes. El trabajo más representativo sobre la representación de la inseguridad lo desarrollaron Guimelli y Rouquette (2004). Estos autores definieron una metodología cualitativa aproximándose al análisis de este fenómeno a través de la versión estructural. Este estudio tenía como finalidad identificar el tipo de relación que pueden presentar los objetos de representación. Para ello, ambos autores plantearon el estudio de la representación social de la inseguridad vs. la seguridad. En los resultados encontraron que ambos objetos son representados con los mismos elementos: la delincuencia, el empleo y salir de noche. Estos elementos permitieron identificar como entre ambos objetos existe una relación antagónica, lo cual permitió corroborar su hipótesis. A pesar de que ambos objetos son contrarios, puesto que la seguridad es un concepto positivo y la inseguridad un concepto negativo, comparten los mismos elementos centrales (Guimelli y Rouquette, 2004).

Por su parte, Umaña (2009) explora la representación que las personas de El Salvador tenían de la inseguridad después de la posguerra. En sus resultados, esta autora encontró que en las zonas de estudio - las cuales eran consideradas objetivamente inseguras-, las personas desarrollaron una visión de pertenencia o naturalización del fenómeno. A pesar de que la población se percataba de la inseguridad, por el hecho de convivir cotidianamente en el contexto, minimizaban los actos delictivos hasta el punto de percibir el entorno seguro. Así también, resulta sumamente interesante que la población posconflicto concibiera la inseguridad como una problemática heredada. Este resultado resaltó tres cosas: la primera, que la inseguridad anterior del conflicto se presentaba en grados menores, por tanto, no era una problemática alarmante; segundo, después del conflicto, a la inseguridad se le otorga un carácter omnipresente, lo cual implica que es un fenómeno presente en el mundo; y la tercera, los efectos que promueven la inseguridad, como por ejemplo la violencia o la delincuencia, también son problemáticas heredadas de la guerra (Umaña, 2009).

La investigación de San Martín (2013) se aproximó al estudio de la representación de la seguridad ciudadana en Chile. Esta indagación es interesante, ya que los elementos evocados por la sociedad chilena al representar la seguridad son negativos (la población evocó elementos referidos solo a la inseguridad). En la representación emergieron elementos tales como delincuencia, inseguridad y miedo. Además, se evocaron elementos relacionados con la incertidumbre y desconfianza social; las personas construyen una visión individualista del contexto en la cual el individuo se protege a sí mismo y a las relaciones próximas, por ejemplo la familia y/o amigos (San Martín, 2013). El trabajo de Guerrero (2007) por su parte, plantea el análisis de la representación social de la inseguridad en Chile, considerando el concepto de segregación social como constructor de representaciones diferenciales. Para ello, este autor realizó una comparación entre dos barrios: uno considerado seguro y otro inseguro. En los resultados, el autor encontró que el posicionamiento social construye representaciones diferenciales. La población afirma que la problemática de la inseguridad tiene su base en el desarrollo social y urbano desigual, así como en la ausencia de un Estado promotor de mejores condiciones de vida (Guerrero, 2007). 


\section{La perspectiva estructural de las representaciones}

La TRs plantea que una representación es el producto de una actividad mental a través de la cual un individuo o un grupo reconstruye la realidad, proporcionándole un significado específico (MinibasPoussard, 2003). Las representaciones sociales son productos imbricados dentro del proceso de la comunicación social (Santana y Chaves, 2009). En este sentido, las representaciones sociales funcionan como mecanismos mediante los cuales se puede conocer la realidad de una sociedad, ya que a través de ellas es posible aproximarse a un conocimiento compartido en el consenso social (Jodelet, 2001).

Esta investigación se centra concretamente en la teoría del núcleo central. Dentro de la TRs, la perspectiva del núcleo central se interesa por el estudio del conocimiento constituyente (proceso), es decir, busca conocer la estructura y la organización de la representación. De esta manera, permite el estudio de las representaciones estables considerando la representación ya no solo como universos de opiniones, sino que las considera también como universos estructurados (Rateau y Lo Monaco, 2013). Asimismo, esta perspectiva plantea que toda representación se encuentra organizada con base en su núcleo central y su sistema periférico (Abric, 2001). Los elementos centrales determinan el significado y la organización de la representación, se caracterizan por ser compartidos en el consenso social y ser inflexibles resistiendo a los cambios. Los elementos periféricos por su parte, son complementarios a los elementos centrales, son flexibles y permiten anclar al sujeto en la realidad cotidiana, a fin de ajustarse a las interacciones y situaciones (Guimelli, 1998). Conocer estos dos sistemas permitirá identificar la estructura y organización de los elementos que mayor saliencia tienen al representar la inseguridad.

\section{Metodología}

\section{Participantes}

Un muestreo por cuotas se utilizó para la inclusión de los participantes. En esta investigación participaron 271 estudiantes universitarios residentes en la ciudad de Ambato. Los participantes fueron jóvenes (67\% mujeres y $33 \%$ hombres), con una media de $21,32$ años ( $\mathrm{DT}=2,23)$.

\section{Instrumento}

El instrumento que se utilizó fue un cuestionario de evocación libre de palabras (Abric, 2001; Vergès, 2003). Este cuestionario fue organizado en dos secciones con el fin de establecer sistemas de complementación entre la estructura y la organización de la representación social. En la primera sección, se solicitaba a cada participante responder las tres primeras respuestas que se le venían a la mente al pensar en la inseguridad. La pregunta inductora se presentó de la siguiente manera: “ ¿Qué se le viene a la mente cuando piensa en la inseguridad?”. Finalmente, en la segunda sección, se solicitaba a cada participante que jerarquizara las respuestas en el orden de importancia que para él/ella tenía cada respuesta.

\section{Procedimiento}

El cuestionario fue aplicado después de una breve presentación del instrumento y de los objetivos del estudio por parte del equipo investigador. La participación en el estudio fue voluntaria y si bien la mayoría de los participantes dio su consentimiento, algunos reusaron participar y dejaron sin responder el cuestionario.

\section{Análisis de los datos}

Las respuestas de los participantes se analizaron mediante el programa Evoc (Programa para el análisis de asociación libre de palabras). Este programa, mediante el análisis lexicográfico, permite organizar las palabras con base en jerarquías tales como frecuencia y orden de evocación (Vergès, 1992; 1994; 2003). A fin de identificar los elementos que son susceptibles de pertenecer al núcleo central, se codificaron las respuestas de los participantes con base en el orden de respuesta (primera respuesta $=1$; segunda respuesta $=2$; etc.). Para ello, se tuvo en consideración la frecuencia intermedia y el rango medio de las palabras evocadas. La frecuencia intermedia establece una segmentación entre las palabras que son muy frecuentes y menos frecuentes en el discurso. El rango medio establece una segmentación del orden de las palabras, es decir, las palabras prioritarias y secundarias en el discurso. Esto permite construir el prototipo representacional (clasificación de las palabras en los cuatro cuadrantes), segmentando los elementos susceptibles de pertenecer al núcleo central y el sistema periférico (Guimelli, 1998). En el núcleo central 
aparecerán los elementos con una alta frecuencia y citados en primer lugar. En la primera periferia (segundo y tercer cuadrante), aparecerán los elementos que continúan teniendo una fuerte frecuencia de evocación y son citados en los primeros lugares. En la segunda periferia (cuarto cuadrante), aparecen las palabras con una baja frecuencia y citados en segundo lugar (Larrañaga, Valencia y Garbiñe, 2012).

Por otra parte, además del análisis lexicográfico, EVOC permite realizar un análisis de categorización con todas las respuestas. Estas categorías permiten centralizar las palabras y así reflejar los diferentes discursos. Permite también identificar las categorías que mayor importancia tienen al representar el objeto de estudio. Con el interés de conocer las relaciones entre los diversos discursos se realizó un análisis de similitud. Este análisis parte de la técnica de análisis de datos basado en la idea de la asociación. De esta manera, es posible destacar la vinculación que los discursos tienen entre sí. Para ello, el análisis de similitud toma como referencia el índice de implicación. Este índice permite conocer el porcentaje de personas que hacen referencia a algún término de una categoría, así como lo hacen también a un término de otra categoría. Estas relaciones se ven expresadas a través de la teoría de grafos. El grafo permite construir una estructura matemática, la cual refleja las distintas relaciones que tienen los discursos (Berge citado en Vergès y Bouriche, 2001). El análisis de implicación se realiza mediante el programa sımi2003 (Vergès, 2001; 2003). Finalmente, se realizó un análisis de comparación entre los hombres y las mujeres a fin de observar las diferencias. Esta comparación se realizó mediante el sub-programa COMPLEX $^{1}$ provisto por el paquete de EVOC (Bonnec, Roussiau y Vergès, 2002).

\section{Resultados}

\section{Prototipo representacional: núcleo central y sistema periférico}

En la pregunta sobre la inseguridad se analizó un $87 \%$ de las evocaciones (palabras que habían sido evocadas al menos cinco veces). Los puntos de corte fueron: frecuencia intermedia $17 \mathrm{y}$ un rango medio

COMPLEX es un sub-programa provisto por el paquete de EVoc (Bonnec, Roussiau y Vergès, 2002). Este permite realizar comparaciones grupales con base en los posicionamientos sociales de interés, como por ejemplo el género, el rol, la edad y el estatus en relación con el objeto de estudio. de 1,9. Los elementos que son susceptibles de pertenecer al núcleo central se encuentran en el primer cuadrante de la tabla 1. Estos elementos refieren tres aspectos sobre la inseguridad. Por un lado, las leyes, las policías, la ciudadanía ${ }^{2}$ y la falta de implementación de leyes hacen referencia a que la inseguridad se debe, en gran medida, a la falta de estructura de las instituciones judiciales (policiacas). Esto es interesante, ya que desde la perspectiva de los jóvenes la falta de agentes en el espacio público genera una percepción de incertidumbre, ya que no perciben protección. Con el fin de ejemplificar dicha afirmación, los jóvenes señalan:

Falta de autoridad policial en zonas de delitos. Deficiente manejo del problema de la inseguridad por autoridades. La falta de policías a partir de las 6 p.m. y falta de resguardo policial en algunas zonas. Falta de leyes y falta de castigos severos. Corrupción por parte del cuerpo policial $^{3}$.

Por otro parte, en el núcleo central aparecen elementos que apoyan el discurso anterior y reflejan la preocupación por la ausencia de policías. Así, las zonas, la probabilidad y el peligro describen la incertidumbre-miedo que los jóvenes sienten al salir a espacios públicos y sentirse desprotegidos. Los jóvenes señalan:

No te sientes tranquilo en un lugar o zona, eres propenso a robos. La falta de resguardo policial en las zonas genera los delitos e inseguridad. No tener la libertad de estar en un lugar o pasar por lugares peligrosos sin compañía. Los robos en ciertas zonas de la ciudad (Subprograma LISTVOC).

Finalmente, el elemento delincuencia viene a señalar que la inseguridad se refleja a través de los robos o los asaltos que las personas pueden padecer en su vida cotidiana. Así, los jóvenes señalan:

\footnotetext{
2 Se introduce ciudadanía en factores estructurales ya que desde la perspectiva de los jóvenes, el problema de la inseguridad y su establecimiento se debe, en parte, a la falta de coordinación entre las instituciones judiciales y los ciudadanos Así, dicha relación puede ayudar a disminuir el problema de la inseguridad.

3 Fuente extraída del sub programa Listvoc provisto por el paquete de Evoc (Bonnec, Roussiau y Vergès, 2002; Vergès, 2003). Este sub programa permite conocer los diferentes discursos que los sujetos priorizan al describir un objeto social, en este caso a la inseguridad.
} 
Hay delincuencia en todo momento y sientes miedo a salir por los robos a personas. Aumento de la delincuencia en la ciudad. Desconfianza en las personas por robos violentos y agresiones físicas. La falta de protección de la policía (Subprograma LISTVOc).

Los elementos que aparecen en la periferia (primer y segundo cuadrante), refuerzan y concretizan los elementos ya señalados en el primer cuadrante o núcleo central. Los elementos agresión, asesinatos, drogas, muertes, atracos, robos, transporte (robo en buses), delitos, secuestro, violaciones y violencia, son ejemplos de los hechos violentos y actos delictivos ligados a la inseguridad, típicos del contexto y que generan incertidumbre. Por su parte, los elementos corrupción y social se refieren a los factores estructurales y ponen de relieve la pobre estructura de las instituciones judiciales. Por otra parte, vigilancia, vulnerabilidad, vivir, desconfianza, miedo, personas, protección, seguridad, calles-libertad (salir), desprotegidos, sentimientos y temor, expresan el miedo e inseguridad (desconfianza) de salir a lugares públicos. Por último, resulta interesante señalar que los jóvenes evocan elementos que estereotipan la figura del delincuente (aspectos negativos) como una persona con baja autoestima e inestabilidad emocional.

En relación con el análisis categorial, se han clasificado las evocaciones en cuatro categorías (ver tabla 2): (I1) Factores estructurales de la inseguridad (e.g. leyes, corrupción); (I2) Miedo e incertidumbre en espacios públicos (e.g. vulnerabilidad, incertidumbre); (I3) Características negativas del delincuente (e.g.

Tabla 1

Frecuencias y rango de evocaciones sobre la inseguridad

\begin{tabular}{|c|c|c|c|c|c|}
\hline & Frecuencia $>17$ & Rango medio $<1,9$ & & Frecuencia $>17$ & Rango medio $>1,9$ \\
\hline $\begin{array}{l}\text { Ciudadana } \\
\text { Delincuencia } \\
\text { Falta } \\
\text { Leyes } \\
\text { Peligro } \\
\text { Policías } \\
\text { Probabilidad } \\
\text { Zonas }\end{array}$ & $\begin{array}{l}20 \\
19 \\
80 \\
20 \\
41 \\
37 \\
18 \\
26\end{array}$ & $\begin{array}{l}1,850 \\
1,842 \\
1,800 \\
1,800 \\
1,463 \\
1,892 \\
1,889 \\
1,846\end{array}$ & $\begin{array}{l}\text { Atracos } \\
\text { Desconfanza } \\
\text { Miedo } \\
\text { Personas } \\
\text { Protección } \\
\text { Robos } \\
\text { Seguridad } \\
\text { Transporte } \\
\text { Violencia }\end{array}$ & $\begin{array}{l}36 \\
43 \\
87 \\
28 \\
22 \\
90 \\
31 \\
25 \\
45\end{array}$ & $\begin{array}{l}2,194 \\
2,000 \\
1,931 \\
2,036 \\
2,000 \\
1,967 \\
2,161 \\
2,120 \\
2,047\end{array}$ \\
\hline & Frecuencia $>17$ & Rango medio $<1,9$ & & Frecuencia $>17$ & Rango medio $>1,9$ \\
\hline Agresión & 15 & 1,667 & Acoso & 5 & 2,400 \\
\hline Asesinatos & 7 & 1,857 & Baja & 10 & 1,900 \\
\hline Autoestima & 13 & 1,846 & Calles & 9 & 2,000 \\
\hline Control & 13 & 1,538 & Casas & 5 & 2,000 \\
\hline Corrupción & 6 & 1,833 & Coraje & 5 & 2,000 \\
\hline Drogas & 6 & 1,875 & Cuidado & 6 & 2,000 \\
\hline Inestabilidad & 14 & 1,889 & Daños & 10 & 2,600 \\
\hline Muertes & 10 & 1,800 & Delincuentes & 12 & 2,286 \\
\hline Social & 9 & 1,667 & Delitos & 6 & 2,000 \\
\hline Vigilancia & 5 & 1,800 & Desprotegidos & 9 & 2,222 \\
\hline Vivir & 5 & 1,400 & Física & 6 & 2,333 \\
\hline \multirow[t]{12}{*}{ Vulnerabilidad } & 14 & 1,643 & Incertidumbre & 7 & 2,286 \\
\hline & & & Intranquilidad & 15 & 2,500 \\
\hline & & & libertad & 7 & 2,286 \\
\hline & & & Golpes & 15 & 2,000 \\
\hline & & & Oportunidades & 6 & 2,167 \\
\hline & & & Prevención & 7 & 2,000 \\
\hline & & & Problemas & 8 & 2,000 \\
\hline & & & Salir & 12 & 2,167 \\
\hline & & & Secuestros & 11 & 2,000 \\
\hline & & & Sentimientos & 15 & 2,067 \\
\hline & & & Temor & 16 & 2,250 \\
\hline & & & Violaciones & 5 & 2,200 \\
\hline
\end{tabular}


autoestima, inestabilidad); y (I4) Hechos violentos y actos delictivos de la inseguridad (e. g. secuestros, robos/asaltos). Las categorías más utilizadas fueron la (I2) Miedo e incertidumbre en espacios públicos, utilizada por un $72 \%$ de los participantes y con un rango intermedio de 2,0; y la (I4) Hechos violentos y actos delictivos de la inseguridad, utilizada por un $61 \%$ con una rango intermedio de 2,0.

Los análisis de similitud realizados con el fin de visualizar la inclusión de los campos semánticos (figura 1), mostraron como todas las categorías tienen una fuerte implicación (más del 40\%) con la categoría central (I2) Miedo e incertidumbre en espacios públicos. Además, a esta categoría se vinculan tres discursos. Por un lado, (I4) Hechos violentos y actos delictivos de la inseguridad tipifican los delitos que cotidianamente se comenten en Ambato, tales como los secuestros, asesinatos, robos o violaciones. Por otro lado, (I1) Factores estructurales de la inseguridad se refiere a la desestructura gubernamental misma, lo cual merma la posibilidad de brindar seguridad ciudadana. Algunos de los elementos de esta categoría se refieren a la falta de leyes y su escasa aplicación de las que existen. Finalmente, (I3) Características negativas del delincuente, estereotipan las características prototípicas que las personas atribuyen al delincuente, tales como, por ejemplo, problemas de autoestima o inestabilidad emocional. Es interesante que sean la categoría (I2) y la (I4) el discurso que destacan los jóvenes. Esto exhibe, por una parte, la preocupación de los individuos de llegar a ser víctimas de un delito, así como la percepción de la probabilidad que tiene su grupo de llegar a ser víctima de un delito. En suma, esto demuestra la percepción de vulnerabilidad que los jóvenes atribuyen a su grupo social.

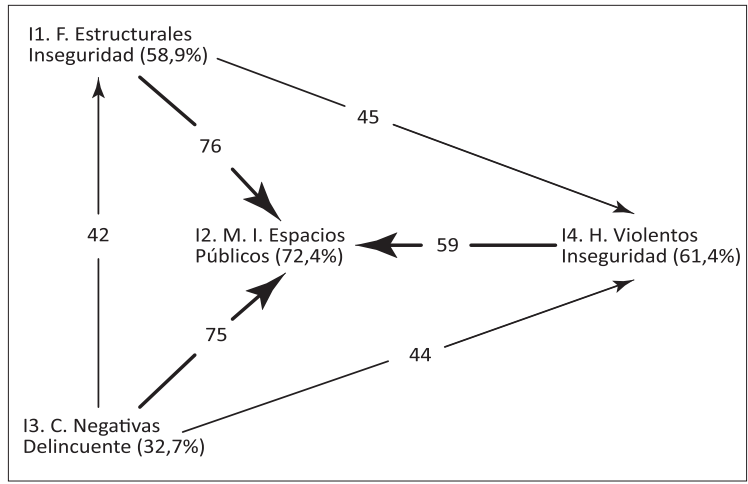

Figura 1. Índice de implicación entre las categorías sobre inseguridad (\%). Elaboración propia.

\section{Comparación de respuestas sobre inseguridad entre hombres y mujeres}

El análisis realizado a través del sub-programa сомPLEX permitió identificar que las respuestas proporcionadas por hombres y mujeres fueron similares. A pesar de ello, se encontró una diferencia significativa

Tabla 2

Categorías sobre la inseguridad

\begin{tabular}{|c|c|c|c|}
\hline Categoría & Campo temático & Palabras principales & Características \\
\hline I1 & $\begin{array}{l}\text { Factores estructurales de la } \\
\text { inseguridad }\end{array}$ & $\begin{array}{l}\text { Falta, leyes, policías, control, corrup- } \\
\text { ción, social, ciudadana, problemas (falta } \\
\text { de estructura) y oportunidades }\end{array}$ & $\begin{array}{l}\text { Señala la pobre estructura del } \\
\text { gobierno y de las instituciones judi- } \\
\text { ciales para proporcionar protección a } \\
\text { la ciudadanía. }\end{array}$ \\
\hline $\mathrm{I} 2$ & $\begin{array}{l}\text { Miedo e incertidumbre en espa- } \\
\text { cios públicos }\end{array}$ & $\begin{array}{l}\text { Probabilidad, protección, zonas, peligro, } \\
\text { coraje, vulnerabilidad, desconfianza, } \\
\text { seguridad, miedo, personas, calles, des- } \\
\text { protegidos, salir, temor, vigilancia, vivir, } \\
\text { cuidado, sentimientos, libertad, preven- } \\
\text { ción, incertidumbre e intranquilidad }\end{array}$ & $\begin{array}{l}\text { Señala la vulnerabilidad e incerti- } \\
\text { dumbre que los ciudadanos sienten al } \\
\text { salir a espacios públicos. Así también, } \\
\text { señala el sentimiento de inseguridad } \\
\text { (desconfianza) presente en la vida } \\
\text { cotidiana de la sociedad. }\end{array}$ \\
\hline I3 & $\begin{array}{l}\text { Características negativas del } \\
\text { delincuente }\end{array}$ & $\begin{array}{l}\text { Autoestima, delincuentes, inestabilidad } \\
\text { y baja }\end{array}$ & $\begin{array}{l}\text { Define el perfil prototipo de un } \\
\text { delincuente. }\end{array}$ \\
\hline I4 & $\begin{array}{l}\text { Hechos violentos y actos delicti- } \\
\text { vos de la inseguridad }\end{array}$ & $\begin{array}{l}\text { Asesinatos, acoso, delincuencia, agre- } \\
\text { sión, delitos, drogas, muertes, atracos, } \\
\text { física (agresiones), robos, transporte, } \\
\text { casas (robo vivienda) violencia, daños, } \\
\text { secuestros, violaciones y golpes }\end{array}$ & $\begin{array}{l}\text { Señalas los hechos violentos típicos } \\
\text { (muertes o secuestros) y característi- } \\
\text { cos de la inseguridad en el contexto } \\
\text { cotidiano. }\end{array}$ \\
\hline
\end{tabular}


que se considera importante señalar. En concreto, es el grupo de las mujeres quienes destacan que sienten más incertidumbre en su vida cotidiana, al hacer referencia al término miedo con mayor frecuencia que los hombres (66 y 21 respectivamente): $t(1141)=1,65$, $p<0,001$. Además, al realizar un análisis en mayor profundidad, se encontraron elementos diferenciales en el discurso de las mujeres. Si bien es cierto que tanto hombres como mujeres hacen referencia a los mismos delitos tales como los robos, las amenazas en la calle o los atracos, y que describen su contexto como inseguro, intranquilo e inestable, es el grupo de mujeres quienes específicamente evocan un discurso de corte emocional característico de la vulnerabilidad (miedo). En concreto, las mujeres refieren elementos tales como la angustia, la desesperación, el estrés, la incertidumbre y los nervios que el contexto de su vida cotidiana les genera. Esta diferencia con base al género se ha encontrado también en estudios previos sobre el miedo al delito (Smith, Torstensson y Johansson, 2001; Tulloch y Jennett, 2001), en los cuales se identificó que las mujeres tienden a sentir más miedo en razón a su grupo de pertenencia que los hombres.

\section{Discusión}

Los resultados del estudio evidenciaron elementos interesantes sobre la dinámica y la estructura de la representación de la inseguridad. En general, el análisis de la representación mostró como desde la perspectiva de los jóvenes, la inseguridad es una problemática que afecta en gran medida su vida cotidiana. Los jóvenes señalan que el hecho de salir a ciertos lugares públicos o zonas específicas, genera un sentimiento de miedo o temor. Este sentimiento tiene su base en el hecho que los jóvenes perciben una alta probabilidad de ser víctimas de algún delito. Bien a un atraco a mano armada, ser acosados en las calles o utilizar el transporte urbano y ser víctimas de un robo violento (Guimelli y Rouquette, 2004; Reyes, Larrañaga y Valencia, 2015; San Martín, 2013).

El hecho de que los jóvenes perciban miedo al salir a espacios públicos ha desarrollado un sentimiento de desconfianza por el desconocido (Vilalta, 2009). En este sentido, al trasladarse de un lugar a otro, este grupo plantea que debe protegerse $y$, por ende, desconfiar de cualquier persona que parezca sospechosa. Del mismo modo, resulta lógico que los jóvenes señalen que es importante conocer quiénes viven en el entorno (barrio y vecindario), ya que el control sobre el vecindario y la organización comunitaria genera un sentimiento de protección y reduce el sentimiento de inseguridad (Umaña, 2009; Vanderschueren et al., 2010). Además, estudios previos realizados mediante la teoría de la desorganización social, muestran como la organización y la existencia de normas en la comunidad reduce la criminalidad comunitaria (Bursik y Grasmick, 1993; Sampson y Stephen, 1999).

Por otra parte, los jóvenes señalan que la inseguridad tiene su base en el hecho de que existe una ineficiente estructura en las instituciones judiciales, incapaz de brindar seguridad, lo cual genera una alta percepción de desprotección. Este resultado es interesante ya que estudios previos encontraron que la población atribuye el problema de la inseguridad a la ausencia de un Estado benefactor; la población exige mejores condiciones para el desarrollo social (Guerrero, 2007; Vite, 2014). Sin embargo, en este estudio los jóvenes, más que un Estado benefactor, demandan un Estado de ley. Los jóvenes señalan que la corrupción existente en la estructura gubernamental $y$, particularmente en la judicial, limita en gran medida combatir la inseguridad. Por un lado, las instituciones policiacas no cumplen su función de velar por la seguridad ciudadana. Por otro, las leyes no se aplican correctamente o se evaden en razón al clientelismo y la burocracia presente en la estructura gubernamental.

Las diferencias de género evidenciaron que las mujeres reportan tener mayor miedo y vulnerabilidad en su vida cotidiana, a diferencia de los hombres, quienes no señalan sentir miedo o temor. Se puede llegar a pensar que las mujeres perciben su grupo de pertenencia con mayores probabilidades de sufrir un delito (Reid y Konrad, 2004; Warr, 2000), o bien que los hombres son más reticentes a demostrar su vulnerabilidad (Gabriel y Grave, 2003; Sutton y Farrall, 2005; Sutton, Robinson y Farrall, 2011).

Finalmente, con base en los resultados encontrados se pueden concluir dos cosas. Primero, el hecho de que en estudios previos la "seguridad" fuese representada mediante elementos negativos (San Martín, 2013), lleva a considerar cómo al estudiar un objeto de representación es necesario tomar en consideración su naturaleza. Objetos ambiguos como la in/seguridad (Guimelli y Roquette, 2004), o como lo han mostrado los estudios sobre paz (Orr, Sagi y Bar-on, 2000; Wagner, Valencia y Elejabarrieta, 1996), pueden generar sesgos en la representación que los sujetos construyen. Por tanto, es importante considerar la naturaleza y definir un método adecuado para aproximarse al análisis de este tipo de objetos. 
Segundo, estos resultados permiten plantear que una línea de indagación muy interesante sería el análisis de la percepción del miedo al delito (Gainey, Alper y Chappell, 2010; Jackson, 2009). El gobierno ha desarrollado estrategias cuyo fin es reducir la inseguridad, tales como los botones de pánico en taxis y transporte público (conectados a la red policial para que apoyen rápidamente ante el robo), la colocación de cámaras en la ciudad y la creación del número de emergencia ECU-911. Sin embargo, estos mecanismos no han ayudado a disminuir la percepción de inseguridad en la ciudadanía, ya que como se encontró en el estudio, desde el discurso de los jóvenes el contexto se percibe inseguro. En este sentido, el estudio del miedo al delito puede identificar si el miedo se establece por el hecho de haber sido víctima de un hecho violento, de manera que se exacerbe la percepción de inseguridad (Vilalta, 2009). Este fenómeno apoyaría en gran medida el desarrollo y el establecimiento de la desconfianza social, la misma que fragmenta las relaciones interpersonales quebrantando en gran medida la sana convivencia y el bienestar de la sociedad.

\section{Referencias}

Abric, J. C. (2001). Prácticas y Representaciones Sociales. México: Ediciones Coyoacán.

Bonnec, Y., Roussiau, N. y Vergès, P. (2002). Categorical and prototipical analysis: A study on the quality-process in hospital institutions. Revue Européenne de Psychologie Appliquée, 52 (3-4), 213-220.

Bursik, R. y Grasmick, H. (1993). The Use of Multiple Indicators to Estimate Crime Trends in American Cities. Journal of Criminal Justice, 21(5), 509-516.

Carrión, F. (1994). Violencia urbana e inseguridad ciudadana: Ecuador. En PNUD/Plan Nacional de Rehabilitación Programa Residencial (Ed.), Memorias del Seminario Internacional "Violencia Urbana e Inseguridad Ciudadana” (pp. 163-168). Bogotá: PNUd /Plan Nacional de Rehabilitación Programa Residencial.

Centro Ecuatoriano de Análisis de Seguridad Integral y Comisión de Estadística de Seguridad Integral. (2013) Estadísticas de seguridad integral noviembre 2013. Recuperado de: http://www.ant.gob.ec/index.php/noticias/estadisticas-de-seguridad-integral-2013.

Gainey, R., Alper., M. y Chappell, A. (2010). Fear of Crime Revisited: Examining the Direct and Indirect Effects of Disorder, Risk Perception, and Social Capital. Am J Crim Just, 36, 120-137. doi: 10.1007/s12103-0109089-8
Gabriel, U. y Grave, W. (2003). The psychology of fear of crime. Conceptual and methodological Perspective. Br J Criminol., 43(3), 600-614.

Garcés, S., Martínez, J., Tite, R. y Tamayo, J. (2014, septiembre). Delitos en la Provincia. Boletín de Coyuntura, 2. Recuperado de: http://www.google.com.ec/ url sa $=t \& r c t=j \& q=\& e s r c=s \&$ source $=$ web \&cd $=1 \&$ ved=0CBwQFjAA\&url=http $\% 3 \mathrm{~A} \% 2 \mathrm{~F} \% 2 \mathrm{Ffca} . u$ ta edu.ec\%2FDocumentos\%2Fobservatorio\%2FBOLETINES\%2520PDF\%2FBOLETIN\%2520No.\%25202. pdf\&ei=UahGVZ6LHsWaNt2kgOAI\&usg=AFQjCNGDJQiO2-avZjT48bc8TNwvsIvZ5A\&bvm=bv.92291466,d.eXY

Guerrero, R. M. (2007). Representaciones Sociales de Inseguridad en dos Comunas de Santiago de Chile. Revista cultura y representaciones sociales, 2(3), 151-168.

Guimelli, C. (1998). Differentiation between the central core elements of social representation: Normative vs. fuctional elements. Swiss Journal of Psychology. 57(4), 209-224.

Guimelli, C. y Rouquette, M. L. (2004). Etude de la relation d'antonymie entre deux objets de représentation sociale : la sécurité vs. l'insécurité des biens et des personnes. Psychologie \& Société, 4(1), 71-87.

Instituto Nacional de Estadística y Censos. [INEC]. (2011). Encuesta de Victimización y Percepción de Inseguridad. Recuperado de: http://www.ecuadorencifras.gob.ec/ documentos/web-inec/Victimizacion/Presentacion_ principales_resultados.pdf.

Jarrín, O. (2005). Políticas Públicas de Seguridad Ciudadana y Proyecto de Ley de Seguridad y Convivencia Ciudadana. Quito, Ecuador: FLAcso.

Jackson, J. (2009). A Psychological Perspective on Vulnerability in the Fear of Crime. Psychology, crime and law, 15(4). 365-390. doi: 10.1080/10683160802275797

Jodelet, D. (2001). Representações sociais: um domínio em expansão. En: Jodelet, D. (Ed.), As representações sociais (pp. 17-44). Rio de Janeiro: EduERJ.

Latinobatómetro. (2013). [Análisis Online país Ecuador]. Datos Duros Inéditos. Recuperado de: http://www. latinobarometro.org/latOnline.jsp.

Larrañaga, M., Valencia, J. y Garbiñe, O. (2012). Efectos de la asimetría de género en la representación social del desempleo femenino. Psykhe, 21(1), 83-98. doi: 10.4067/ S0718-22282012000100006

Minibas-Poussard, J. (2003). Les représentation sociales sur l'argent, la banque et l'épargne. Paris: GREgor IAE de Paris, Université Paris 1 Pantheon Sorbonne. 
Núñez, J. (2011). Crítica a la seguridad ciudadana en Ecuador, 91 estrategias contra la violencia. Quito: FLACso.

Orr, E., Sagi, S. y Bar-On, D. (2000). Social representations in use: Israeli and Palestinian high school students collective coping and defense. Papers on Social Representations, 9, 2.1-2.20. Recuperado de: http://www.psych. lse.ac.uk/psr/PSR2000/9_2Orr.pdf

Palomeque, E. (2002). Diagnóstico sobre seguridad ciudadana en el Ecuador. En F. Carrión (Ed.), Seguridad ciudadana, ¿espejismo o realidad? (pp. 235-249). Quito, Ecuador: FLACSO.

Ramírez, F. y Ágora Democrática (2011). Primera Encuesta Nacional sobre Jóvenes y Participación Política en Ecuador. Recuperado de: http://www.activate.ec/content/primera-encuesta-nacional-sobre-jovenes-y-participacion-politica-en-ecuador.

Rateau, P. y Lo Monaco, G. (2013). La teoría de las representaciones sociales: orientaciones conceptuales, campos de aplicaciones y métodos. Revista CEs Psicología, 6(1), $22-42$.

Reid, L. W. y Konrad, M. (2004). The gender gap in fear: Assessing the interactive effects of gender and perceived risk on fear of crime. Sociological Spectrum, 24(4), 399425. doi: 10.1080/02732170490431331

Reyes, H., Larrañaga, M. y Valencia, J. (2015). Dependencia representacional entre dos objetos sociales: El narcotráfico y la violencia. Cultura y representaciones sociales, 9(18), 162-186.

Sampson, R. y Stephen, R. (1999). Systematic Social Observation of Public Spaces: A New Look at Disorder in Urban Neighborhoods. American Journal of Sociology, 105(3), 603-651.

San Martín, C. (2013). Las Representaciones sociales de la seguridad ciudadana en los vecinos de la comuna de Melipilla, Chile. Psicoperspectivas, 12(1), 72-94.

Santana, M. S. y Chaves, E. M. (2009). Atividade Física e Bem-Estar na Velhice. Revista salud pública, 11(2), 225-239. doi: 10.1590/S0124-00642009000200007

Smith, W. R., Torstensson, M. y Johansson, K. (2001). Perceived risk and fear of crime: Gender differences in contextual sensitivity. International Review of Victimology, 8, 159-181. doi: 10.1177/026975800100800204

Sutton, R. y Farrall, S. (2005). Gender, socially desirable responding, and the fear of crime: Are women really more anxious about crime? British Journal of Criminology, 45(2), 212-224. doi: 10.1093/bjc/azh084.

Sutton, R. M., Robinson, B. y Farrall, S. D. (2011). Gender, fear of crime, and self-presentation: An experimental investigation. Psychology, Crime and Law, 17(5), 421433.
Tulloch. M. y Jennett, C. (2001). Women's responses to fear of crime. Security Journal, 14, 53-62. doi:10.1057/palgrave.sj.8340082

Umaña, L. (2009). Representaciones sociales de la inseguridad en El Salvador de la posguerra: Estudio de casos del Amss. Revista Realidad 120, 389-418.

Vanderschueren, F., Olave, R., Ruiz, J., Mosciatti, E., Del Canto, C. y Díaz, G. (2010). Guía para la prevención en barrios. Hacía políticas de cohesión social y seguri dad ciudadana. Recuperado de: http://www.google.es/ url? sa =t\&rct=j\&q=\&esrc=s\&source $=$ web \& $c d=1 \&$ ved=0CCIQFjAA\&url=http $\% 3 \mathrm{~A} \% 2 \mathrm{~F} \% 2$ Funhabitat org\%2F\%3Fwpdmact\%3Dprocess\%26did\%3DM zg2LmhvdGxpbms\%3D\&ei=o5ArVfH6M4mOLL_ gLAD\&usg=AFQjCNEPsANJtoR1Esc3sucU2bLkJserqQ\&sig2=v78qegJeq8C9jizFJDz9Qw\&bvm= bv.90491159,d.ZWU\&cad=rja

Vergès, P. (1992). Lévocation de l'argent : une méthode pour la définition du noyau central d'une représentation. Bulletin de Psychologie. XLV, 405, 203-209.

Vergès, P. (1994). Approche du noyau central: propriétés quantitatives et structurales. En C. Guimelli (Ed.) Structure et transformations des représentations sociales (pp. 233-253). Neuchâtel: Delachaux et Niestlé.

Vergès, P. (2001). Lanalyse des représentations sociales par questionnaires. Revue française de sociologie, 42(3), 537-561.

Vergès, P. (2003). Ensemble de programmes permettant l'analyse des évocations, evoc2000 [CD Rom]. Aix en Provence, France: Laboratoire Méditerranéen de Sociologie.

Vergès, P. y Bouriche. (2001). L'analyse des données par les graphes de similitude. Recuperado de http://www. scienceshumaines.com/textesInedits/Bouriche.pdf

Vilalta, C. J. (2009). El miedo al crimen en México. Estructura lógica, bases empíricas y recomendaciones iniciales de política pública. Gestión y política pública. 19(1), 3-36.

Vite, M. A. (2014). Reflexiones sobre la violencia y vulnerabilidad en México. Espiral, Estudios sobre Estado y Sociedad, 21(61), 227-258.

Warr, M. (2000). Fear of crime in the United States; Avenues for Research and Policy. En D. Duffee (Ed.), Criminal justice 2000, Vol. 4: Measurement and analysis of crime and justice (pp 451-490). Washington: National Institute of Justice.

Wagner, W., Valencia, J. y Elejabarrieta, F. (1996). Relevance, Discourse and the "Hot" Stable Core of Social Representations. British Journal of Social Psychology, 35(3), $331-351$ 